\title{
Identificación y caracterización del agente causal de la pudrición de la raíz y tallo del maíz (zea mays 1.)
}

\author{
Identification and characterization of the causal agent of the rot of root and \\ stem of maize (zea mays 1.)
}

\section{Milly Minaya Huerta ${ }^{1}$ y Eusebio Reyes Huamán ${ }^{1}$}

\section{RESUMEN}

El objetivo de la presente investigación fue identificar y caracterizar el agente causal de la pudrición de la raíz y el tallo del maíz. Se ejecutó en dos fases: en campo y en laboratorio. El muestreo se hizo en la provincia de Yungay, localidades de Mancos, Ranrahirca, Huarascucho, Yungay y Cañasbamba. La muestra fue de cinco parcelas por localidad, y cinco muestras por parcela; con un total de 125 muestras entre raíces y tallos; las muestras fueron procesadas mediante las técnicas de cultivo en medio Papa Dextrosa Agar Oxitetraciclina-PDAO. También se procesó semillas de maíz obtenidas de comerciantes con la técnica de cámara húmeda. Desarrolladas las colonias de hongos en el medio de cultivo PDAO se procedió a hacer montajes microscópicos de las estructuras y su caracterización e identificación haciendo uso de claves taxonómicas incluidas en la bibliografía. De las muestras de campo se aisló el hongo Fusarium moniliforme en 60\%; también se aislaron Exserohilum turcicum, Nigrospora oryzae, Rhizoctonia solani, y otros hongos de menor importancia: Phoma sp, Penicillium sp, y Bipolaris maydis. En las muestras de semillas se encontró: Cladosporium sp. 53 \%, Fusarium moniliforme 35 \%, Phoma sp. 35 \%, y Fusarium sp. 9\%, Concluimos que Fusarium moniliforme es el agente causal principal de la pudrición de la raíz y tallo del maíz en el Callejón de Huaylas.

Palabras clave: pudrición; raíz; tallo; maíz; identificación.

\footnotetext{
1 Universidad Nacional «Santiago Antúnez de Mayolo» .Huaraz, Perú.
} 


\begin{abstract}
The objective of this research was to identification and characterization the causal agent of the rot of the root and stem of maize running in two phases: in the field and in the laboratory. Sampling was conducted in the province of Yungay in locations; Mancos, Ranrahirca, Huarascucho, Yungay and Cañasbamba, five plots were sampled by locality making a total of 25 and five samples per plot totaling 125 samples between the roots and stems, then led to the Plant Pathology Laboratory of the Faculty of Agricultural Sciences of the UNASAM, and were processed by the cultivation techniques in a papa dextrose agar oxytetracycline-PDAO, also were processed maize seeds obtained from traders with the technique of humid chamber. Once developed colonies of fungi in the culture medium PDAO proceeded to make microscopic mounts of the structures and proceeded to its characterization and identification by making use of taxonomic keys listed in the bibliography. The field samples was isolated the fungus Fusarium moniliforme en 60\%; it also isolated Exserobilum turcicum, Nigrospora oryzae, Rhizoctonia solani, and other fungi of minor importance: Phoma sp, Penicillium sp, and Bipolaris maydis. In the seed samples of found: Cladosporium sp. $53 \%$, Fusarium moniliforme $35 \%$, Phoma sp $35 \%$, and Fusarium sp. $9 \%$, concluding that Fusarium moniliforme is the main causal agent of the rot of the root and stem of maize in the Callejón de Huaylas.
\end{abstract}

Keyswords: rot; root; stem; maize; identification.

\title{
ICHIKLLACHAW
}

Kay musyapakuywanqa riqiqan imanawmi harapa watsinwan tullun ismunqantam. Kayqa chakrachawwan laburaturyu nishqanchawmi rurakashqa. Kay musyapakuyqa rurakashqa Mankuschaw, Waraskuchuchaw, Yunkaychaw Kañaspampachaw, kay llapan markakuna kayan Yunkay suyuchaw. Tsaypaq kay markakunachaw hukllayllapayan parsilakunachaw hurqaqashqa, tsay llapan 25piqwan pitsqa parsilawan; tsaypaq 125 watsiwan tulluchawmi rurakashqa; tsaypiqnam UNASAMpa Ciencias agrariapa Facultaaninman Fitopatuluhiyaman apakashqa, kaychawnam rurakashqa imanaw murukuynin kanqanta papa Diktrusa Agar Oxitetracilina-PDAO nishqanchaw. Hinaman hara murukunata tikrakashqa rantikuqkunapiq rantiikur uqushqa kamara tiknika nishqanwan. Kay quchu aputukuna wiñariyaptin murukuy PDAO nishqanchaw ñawillawanqa mana rikakuq imanaw kanqan rurakanqanta. Chakrachaw rurakashqawan rakikarqan kay ichik ñawillawanqa mana rikakuq kurukunata Fusarium moniliforme 60\%chaw; hinaman rakikashqa Exserobilum turcicum, Nigrospora oryzae, Rhizoctonia solani nishqankuna, hinamanpis ichik kaqkunatapis: Phoma sp, Penicillium sp, y Bipolaris maydis nishqankuna. Kay murukunawan uryashqachaw tarikashqa Cladosporium sp. 53 \%, Fusarium moniliforme 35 \%, Phoma sp. 35 \%, y Fusarium sp. 9\% kanqantam. Ichik shimillachaw nishwan Waylas Raqrachaw Fusarium moniliforme nishqanmi harakunapa watsintawan tullunta ismutsin.

Pushaq shimikuna: Ismuynin; watsi; tullun; hara; imanaw kanqan. 


\section{INTRODUCCIÓN}

El maíz (Zea mays L.) es una especie clave en la alimentación humana y animal. Debido a su gran productividad y adaptabilidad, se cultiva en todo el planeta, y se desarrolla en todos los continentes; a nivel mundial ocupa el tercer lugar después del trigo y el arroz; en la Región Ancash ocupa el primer lugar en cuanto a área cultivada.

El maíz, como cualquier otro cultivo, está expuesto en la naturaleza a una serie de factores adversos, tanto biológicos como ambientales. Entre muchos patógenos que infectan a la planta del maíz, el que causa la enfermedad conocida como «pudrición del tallo» reviste particular interés por su amplia distribución, especialmente en áreas tropicales y subtropicales, su alta capacidad patogénica y de sobrevivencia, así como por su amplio rango de especies de plantas hospedantes. La presencia de esta especie y sus toxinas en los granos representa un problema de primer orden para la industria del maíz por las enormes implicaciones que tiene tanto en la cantidad cosechada, calidad del grano así como en la salud humana y animal.

En el Callejón de Huaylas se observa un problema patológico en el cultivo de maíz, el cual se manifiesta con la pudrición de raíces y tallos; se presenta con mayor severidad en el estado de floración con la disminución de la cantidad y calidad en el rendimiento, lo cual causa preocupación en los agricultores; inicialmente se ha presentado en la provincia de Yungay en parcelas de maíz para producción de choclo, pero no se ha determinado el agente causal de esta enfermedad.

En cuanto a antecedentes sobre enfermedades similares, Agrios (2002), reporta una enfermedad en el maíz llamada podredumbre seca, causada por Strenocarpella maydis; las plantas afectadas mueren repentinamente, las hojas se marchitan, se secan y toman un color verde grisáceo semejante al provocado por el daño de helada. Los internodios (entrenudos) inferiores se tornan de marrones a pajizos, esponjosos y fácilmente quebradizos. La médula se desintegra y pierde color aunque permanecen intactos sólo los haces vasculares.

Por otro lado, Sarasola (1975), menciona una enfermedad en el maíz a la que denomina podredumbre de la raíz y de la base del tallo, causada por Gibberella zeae, cuya fase asexual es Fusarium graminearum; mencionan que se caracteriza porque las hojas en las plantas precozmente infectadas se tornan en forma brusca verde-grisáceas opacas, los tallos muestran con frecuencia en la zona enferma, una decoloración interna rosa o rojiza y fragmentación de la médula.

Según Durán (2007) y Latorre (1999), en el marchitamiento el maíz, seguido de una pudrición, esta podredumbre es difícil de distinguir de la podredumbre del tallo causada por Gibberella. La enfermedad es causada por Fusarium moniliforme, cuya fase sexual es Gibberella fujikuori, que afecta en general las raíces, la base de la planta y los entrenudos inferiores. La podredumbre se presenta desde el inicio de floración y los daños son más severos a medida que la planta madura. 
Moya y Apablaza (2004), utilizando semillas asépticas, partidas en la mitad del embrión, y enteras sin desinfectar, fueron colocadas en medio del cultivo PDA, incubado entre $23^{\circ}$ y $25^{\circ} \mathrm{C}$ por una semana, encontraron que estaban infectadas con Fusarium moniliforme.

El propósito de este trabajo de investigación fue conocer el microorganismo patógeno causante de la pudrición de la raíz y tallo del maíz, para implementar métodos de control o manejo adecuado de la enfermedad, dado que causa enormes bajas en los rendimientos, principalmente en la producción de maíz para choclo. Por lo que los objetivos de este trabajo fueron: identificar y caracterizar el agente causal de esta enfermedad en el Callejón de Huaylas.

\section{MATERIALES Y MÉTODOS}

La presente investigación es de tipo cualitativo, por tratarse de la descripción de las características del causante de la enfermedad; por lo que no se adecúa a un diseño estadístico usado principalmente en una investigación cuantitativa.

El ámbito de muestreo en campo se realizó en la provincia de Yungay, en las localidades de Mancos a $2507 \mathrm{msnm}$, Ranrahirca a $2451 \mathrm{msnm}$, Huarascucho a $2451 \mathrm{msnm}$, Yungay a $2548 \mathrm{msnm}$ y Cañasbamba a $2400 \mathrm{msnm}$. La fase de laboratorio se llevó a cabo en los ambientes del Laboratorio de Fitopatología de la Facultad de Ciencias Agrarias de la UNASAM, ubicado en la Ciudad Universitaria de Shancayán. Se evaluaron cinco campos por localidad, y cinco muestras por campo o parcela, ya que en las observaciones previas se observó que la sintomatología era muy similar.

Para el muestreo se recorrieron en zigzag las parcelas seleccionadas, observando y registrando minuciosamente los síntomas y/o signos de la enfermedad en raíces y tallos. Se tomó muestras de todas estas partes de la planta que mostraban los síntomas típicos de la enfermedad; se las colocó en bolsas de polietileno se las identificó con la información necesaria y fueron llevadas al laboratorio de Fitopatología.

Las muestras fueron lavadas con agua de caño hasta eliminar la tierra adherida, todas las impurezas y/o contaminantes posibles; luego se tomó pequeñas submuestras, las que luego fueron desinfectadas con hipoclorito de sodio al $0.5 \%$, por 3 a 5 minutos; seguidamente se enjuagaron con agua destilada hasta por cinco veces para eliminar el hipoclorito; luego se las pasó a papel absorbente, para eliminar el exceso de agua.

Con una pinza desinfectada se colocó de 3 a 5 muestras por placa de Petri que contenían medio PDAO y se las dejó incubando en la cámara aséptica a una temperatura entre 18 a $22^{\circ} \mathrm{C}$ con una fuente de luz para incentivar la esporulación. Para determinar si la enfermedad también infecta a las semillas, se adquirió muestras para procesarlas en cámara húmeda. Una vez esporulado el patógeno, tanto de la cámara húmeda como del medio de cultivo PDAO, se hizo repiques o resiembras a nuevas placas con medio de cultivo, teniendo en cuenta las características del desarrollo de las colonias para purificar o separar los aislamientos. 
Cuando las colonias purificadas habían desarrollado, se procedió a hacer montajes en láminas porta y cubreobjetos en una gota de lactofenol en azul de metileno utilizando un estilete. Para ver la formación de clamidosporas, se tomó porciones de micelio y se las colocó en $10 \mathrm{ml}$ de agua destilada estéril y se dejaron incubando a temperatura ambiente. Cada ocho días se tomó pequeñas muestras de micelio y se preparó montajes para las observaciones microscópicas, hasta por 32 días para constatar la presencia o ausencia de clamidosporas.

\section{RESULTADOS}

1. Síntomas en raíces. La enfermedad presenta una pudrición de las raíces secundarias, en la zona de los tallos cubiertos por el aporque, también se observan estrías necróticas seguidas de pudrición. En las raíces secundarias aparentemente sanas se inicia una proliferación de raíces terciarias, que posteriormente también son atacadas por el patógeno. Cuando las raíces son dañadas por insectos fitófagos (gusanos de tierra), la pudrición es más severa (Figura 1).
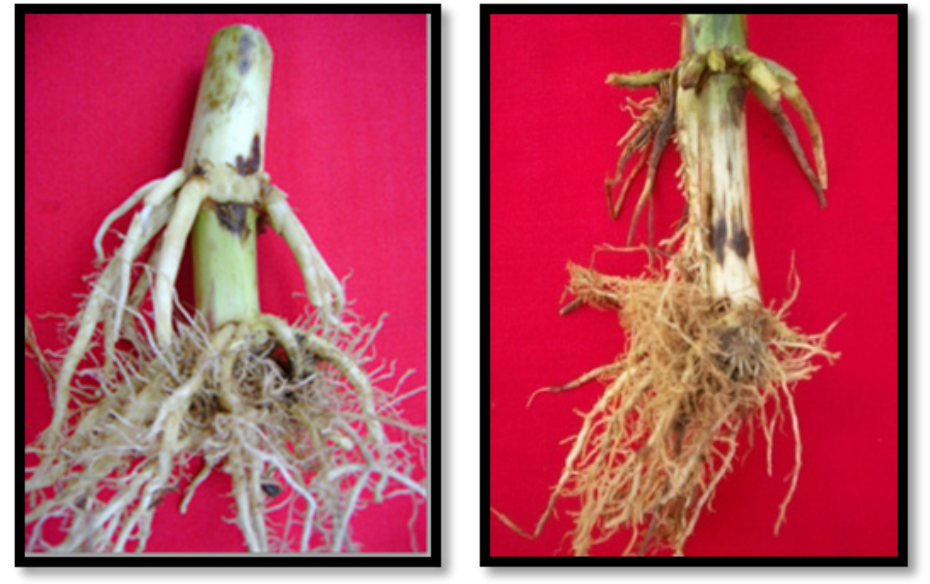

Figura 1. Pudrición de raíces secundarias y estrías necróticas en la base de los tallos

2. Síntomas en tallos. Se manifestaron en los entrenudos, nudos y las vainas de las hojas. En la base de la vaina se inicia una pudrición que la destruye y en los entrenudos se observa manchas y estrías necróticas de color marrón oscuro seguidas de pudrición.

3. Síntomas en hojas. Las hojas de plantas afectadas se muestran cloróticas amarillentas, seguidas de necrosis apical y por los bordes; además muestran una ligera marchitez. En las hojas superiores se inicia una necrosis apical que avanza hacia abajo (Figura 2). Algunas plantas no forman mazorcas y las que llegan a formar, resultan pequeñas y deformes. 

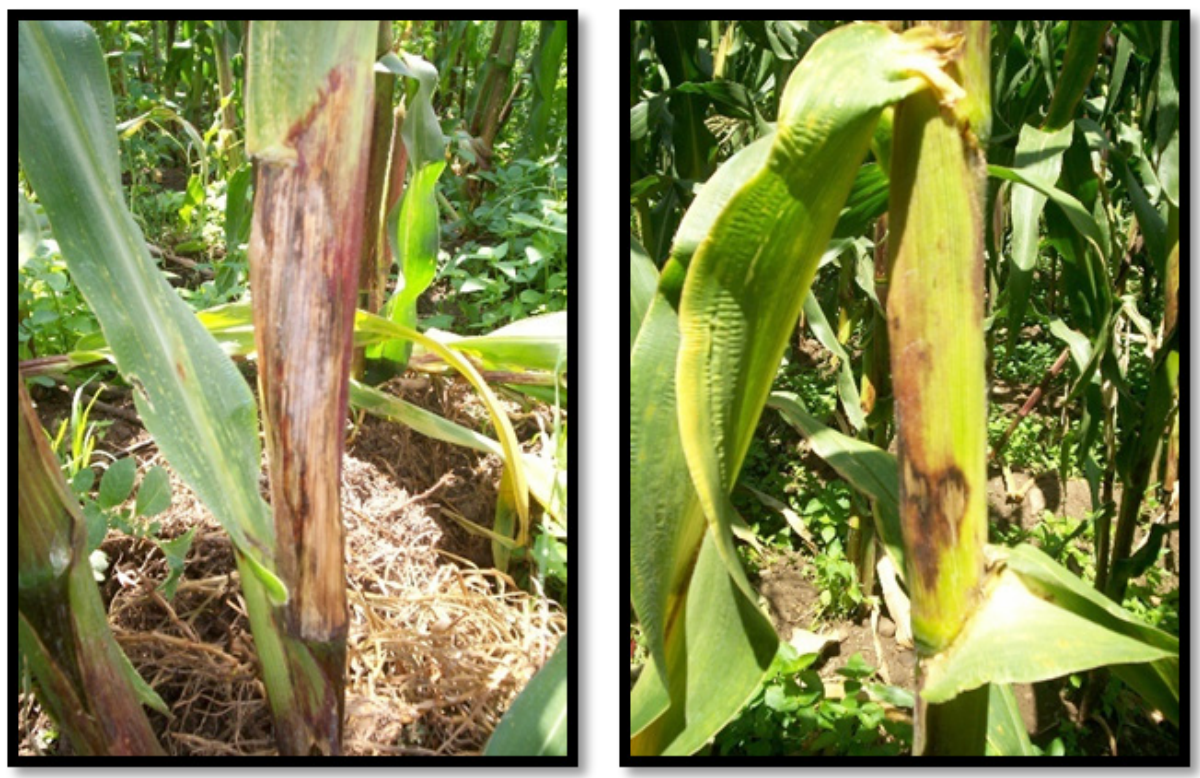

Figura 2. Amarillamiento y pudrición de la vaina de la hoja

Cuando la enfermedad avanza y se vuelve más severa, las vainas se pudren y las hojas se tornan amarillas y posteriormente se secan. En un estado avanzado de la enfermedad y en condiciones de alta humedad, en la cara externa e interna de las vainas de las hojas se observa manchas y estrías necróticas de color marrón oscuro, de diferentes formas y tamaños, dando el aspecto de una piel de felino (figura 3).
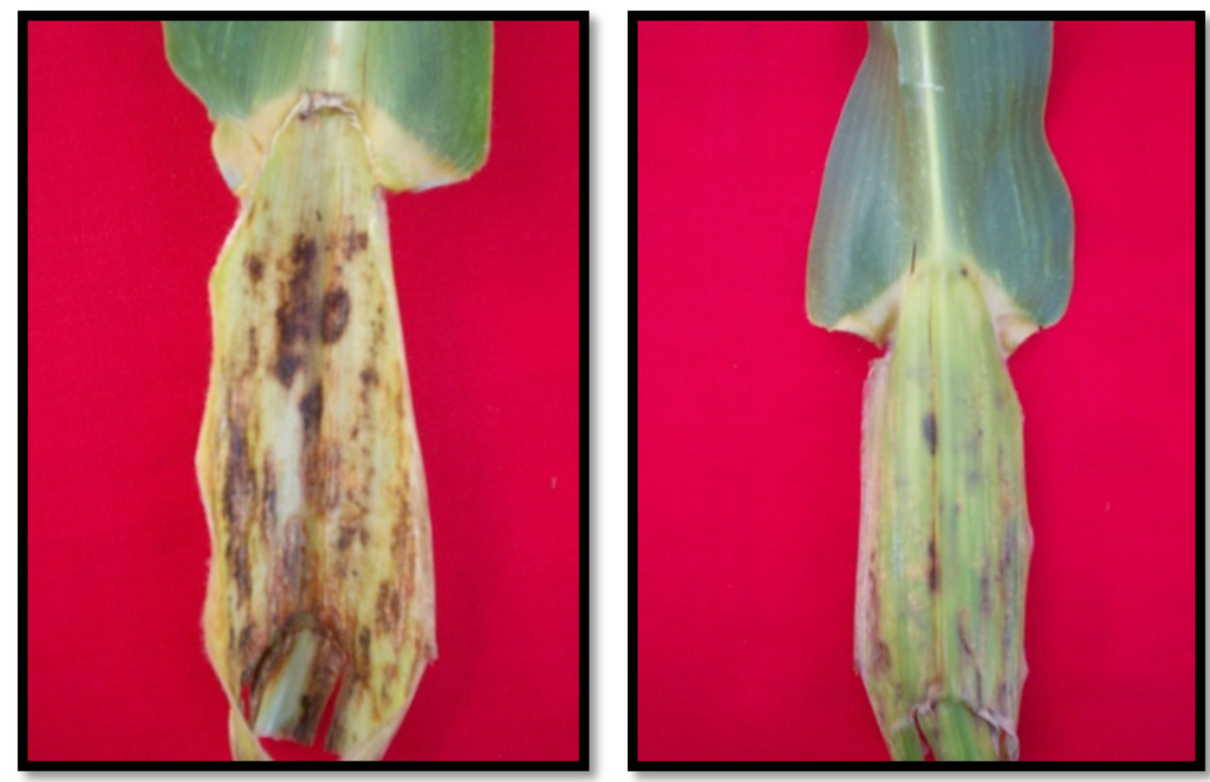

Figura 3. Síntomas producidos en las vainas dando la apariencia de una piel de felino 
4. Síntomas en las brácteas de las mazorcas. Se inician con manchas necróticas, y cuando las plantas son aplicadas con algún producto químico se detiene el avance de la necrosis dando la apariencia de haber madurado el choclo. Cuando no se aplica ningún producto la necrosis se generaliza iniciándose el secado de las brácteas externas y los granos son desuniformes.

\section{Caracterización de los aislamientos en el laboratorio}

Aislamiento 1. Presentó un micelio superficial de color rosa salmón en el medio de cultivo PDAO. El desarrollo de las colonias fue semialgodonosa al inicio; al pasar el tiempo el hongo segrega un pigmento que torna al medio de cultivo de color melón (Figura 4).

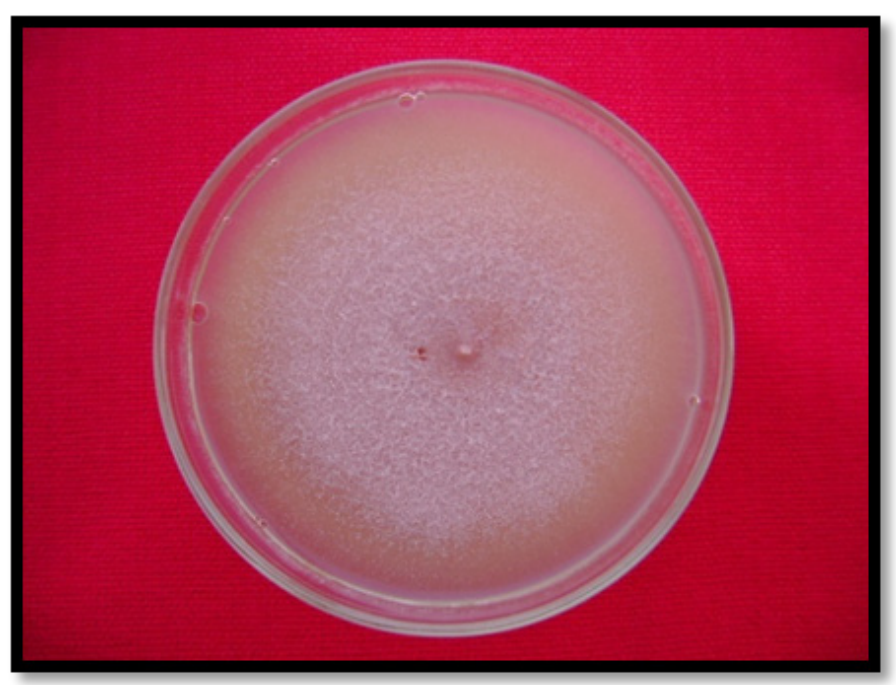

Figura 4. Colonia de Fusarium moniliforme en medio PDAO

Al microscopio se observó un micelio hialino, septado, del cual nacen los conidióforos, que pueden ser cortos o alargados, ramificados o simples, de ellos emergen las microconidias y macroconidias que son hialinas (Figura 5). En las observaciones microscópicas del micelio incubadas en agua destilada estéril, no se observó la formación de clamidosporas. 


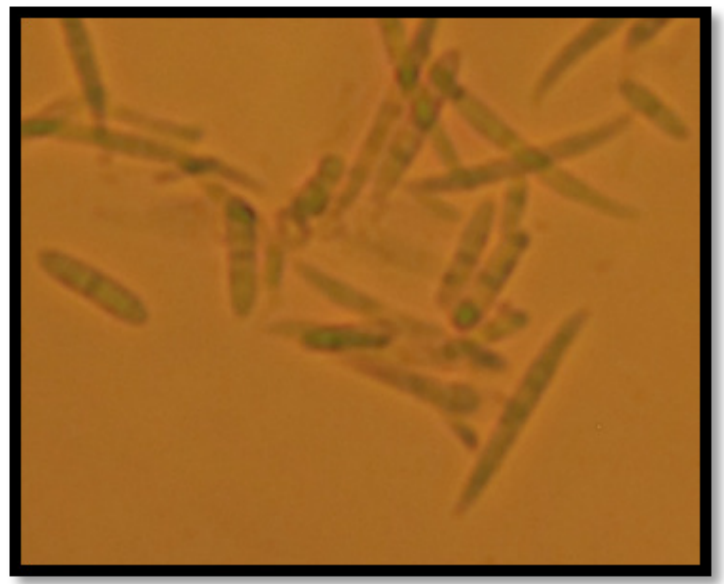

Figura 5. Macroconidias y microconidias de Fusarium moniliforme alargadas y angostas

Aislamiento 2. Presentó un micelio oscuro, septado, desarrollo superficial sobre los medios de cultivo, conidióforos individuales o en clúster, largos, erectos, de color marrón. Conidias del tipo porosporas o sea que se desarrollan lateralmente a través de poros. Las conidias son de color oliváceo, con tres a ocho septas y forma de huso con un hilum prominente (Figura 6).

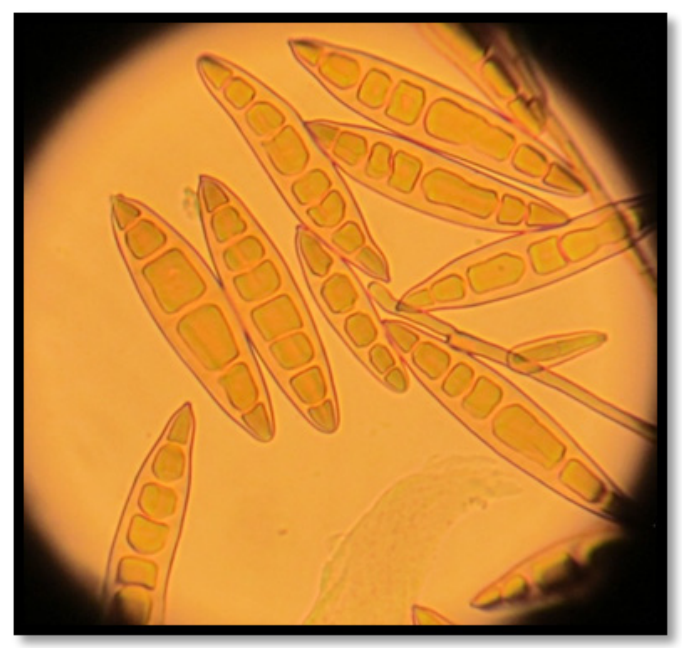

Figura 6. Conidias de Exserohilum turcicum

Aislamiento 3. Micelio blanquecino, desarrollo superficial algodonoso en medio de cultivo PDAO. Conidias oscuras a negras, redondeadas- globosas unicelulares, se desarrollan sobre una vesícula hialina ubicada en la parte terminal de los conidióforos cortos (Figura 7). Este hongo fue aislado principalmente de vainas y hojas de las muestras de campo. 


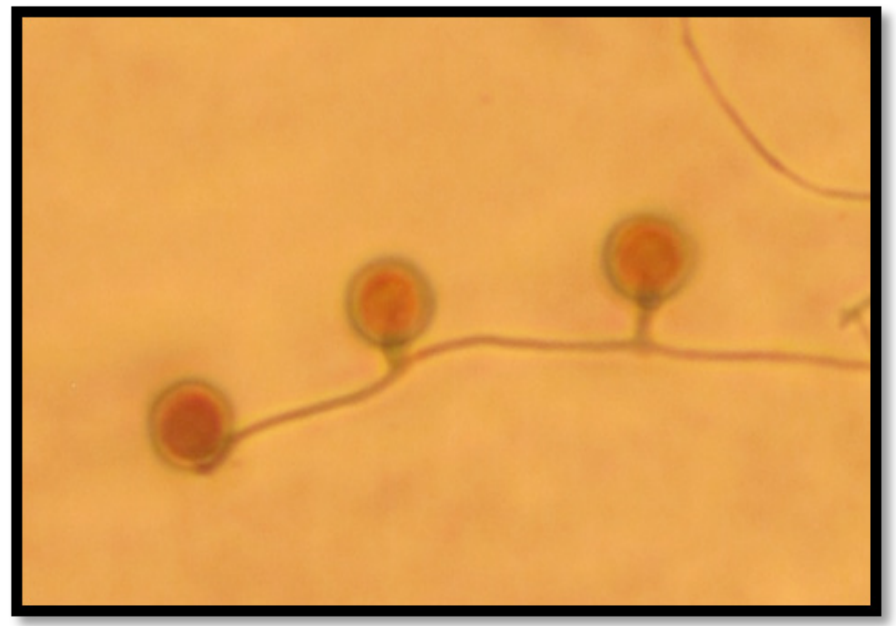

Figura 7. Conidióforos y conidias oscuros de Nigrospora oryzae

Aislamiento 4. Las colonias son de crecimiento rápido, y torna el medio de color marrón. No forman estructuras fructificantes (esporas, conidios). Presenta un micelio grueso marrón, decrecimiento rápido, septado, ramificación en ángulo recto, con una ligera depresión en la base de la ramificación (Figura 8).

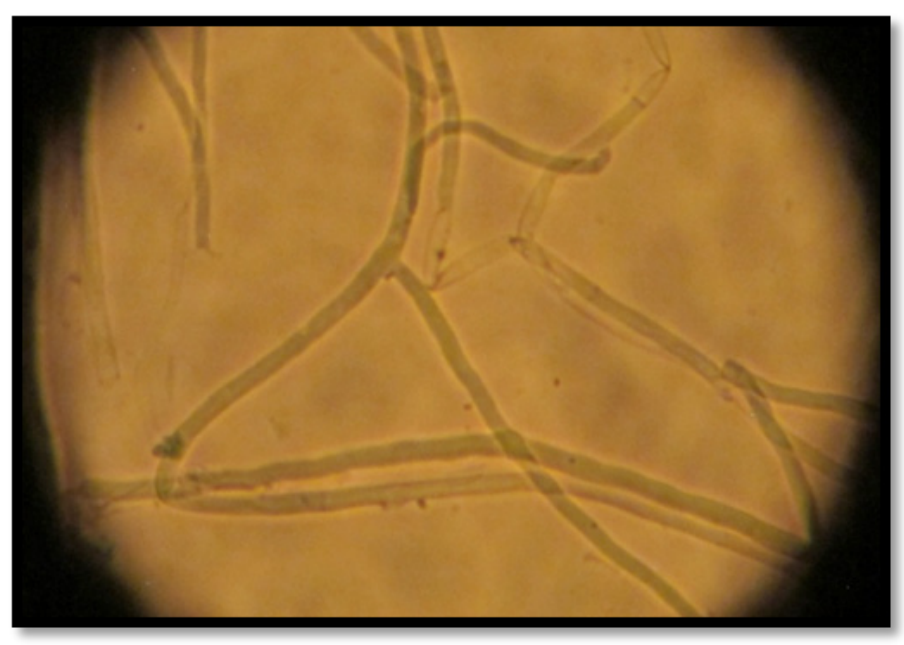

Figura 8. Vista microscópica del micelio de Rbiroctonia solani

Otros aislamientos. De las muestras de campo también se aislaron los hongos: Phoma sp. Penicillium spp. y Bipolaris maydis. 
Tabla 1. Frecuencia de los microorganismos aislados

\begin{tabular}{ccccc}
\hline $\begin{array}{c}\text { Número } \\
\text { de clases }\end{array}$ & Agente causal & $\begin{array}{c}\text { Frecuencia } \\
\text { absoluta }\end{array}$ & $\begin{array}{c}\text { Frecuencia } \\
\text { relativa }\end{array}$ & $\begin{array}{c}\text { Frecuencia } \\
\text { porcentual }\end{array}$ \\
\hline 1 & Fusarium moniliforme & 75 & 0.60 & 60 \\
2 & Exserobilum turcicum & 15 & 0.12 & 12 \\
3 & Nigrospora oryzae & 10 & 0.08 & 8 \\
4 & Rhizoctonia solani & 10 & 0.08 & 8 \\
5 & Otros & 15 & 0.12 & 12 \\
\hline
\end{tabular}

6. Evaluación de semillas en cámara húmeda. De las 130 semillas evaluadas en cámara húmeda se obtuvo los siguientes microorganismos fungosos: Fusarium moniliforme en 46 semillas que corresponde a 35\%; Phoma sp., en 17 semillas afectadas 13\%; Fusarium sp. en 13 semillas equivalente a 9\% y Cladosporium sp., en 69 semillas correspondiente a 53\% del total de semillas evaluadas. En los resultados se observa que Cladosporium sp; es el microorganismo que mayormente se encontró en las semillas; sin embargo este género contiene especies principalmente saprófitas según la literatura consultada (Barnett y Hunter, 1999).

Tabla 2. Hongos presentes en semillas de maíz sembradas en cámara húmeda.

\begin{tabular}{ccc}
\hline Hongos & \multicolumn{2}{c}{ Cámara húmeda } \\
No de semillas afectadas & $\begin{array}{c}\text { Porcentaje }(\%) \\
\text { de semillas afectadas }\end{array}$ \\
\hline Phoma sp. & $17^{\mathrm{a}} / 130^{\mathrm{b}}$ & $13 \%$ \\
Fusarium moniliforme & $46 / 130$ & $35 \%$ \\
Cladosporium sp & $69 / 130$ & $53 \%$ \\
Fusarium sp & $12 / 130$ & $9 \%$ \\
\hline
\end{tabular}

${ }^{a}$ Número de semillas infectadas.

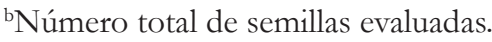

\section{DISCUSIÓN}

La sintomatología que presenta esta enfermedad, las características morfológicas y la no formación de clamidosporas del primer aislamiento coinciden con los reportados por Durán (2007) y Latorre (1999), Moya y Apablaza (2004) para Fusarium moniliforme. Esta enfermedad se ha convertido en un problema debido al monocultivo del maíz para choclo. Exserobilum turcicum y Bipolaris maydis son patógenos netamente foliares, pero cuando hay necrosis en cualquier parte de la planta también se desarrollan, tal como lo menciona Agrios (2002). Rhizoctonia solani es un patógeno de suelo, pero es poco patogénico en este cultivo según Latorre (1999), por lo que no se le puede atribuir ser el causante directo de la pudrición de la raíz y tallo del maíz. Según la tabla 1 , de las 125 muestras analizadas entre raíz y tallo en setenta y cinco de éstas se encontró el aislamiento 01 que corresponde al Fusarium moniliforme en $60 \%$ del total de las muestras. Esto evidencia que este hongo es el principal causante de la pudrición de la raíz 
y tallo del maíz. Exserobilum turcicum se encontró en un 12\%, principalmente en vainas y tallos; dado que este patógeno es causante de manchas foliares en hojas de maíz. Lo que significa que el patógeno puede sobrevivir de una campaña a otra y debe ser tomado en cuenta para el manejo de la enfermedad Nigrospora oryzae se encontró en un $8 \%$ de las muestras analizadas al igual que Rhizoctonia solani; estos patógenos también están reportados en la literatura que afectan al maíz (Latorre, 1999; White, 2004)); sin embargo están considerados como patógenos secundarios, pero que al formar el complejo fungoso las pudriciones de la raíz y tallo los daños son más severos. Y con menos frecuencia se aislaron Phoma sp., Penicilliums pp., Bipolaris maydis; en cinco muestras, cada una equivalente a un $4 \%$ de las muestras analizadas, respectivamente. Según la literatura reportada estos hongos siempre se encuentran asociados al maíz, pero actúan como microorganismos secundarios (White, 2004; Sutton, 1980). En la tabla 2 se muestra que Fusarium moniliforme está en un 35\% de las semillas analizadas, lo que indica que esta especie es diseminada también a través de la semilla a los campos definitivos, causando pudriciones en la raíz, tallo y otros órganos aéreos cuando las condiciones son favorables. Además se encontraron Phoma sp. en un 13\% y Fusarium sp. en un 9\%. Estos resultados concuerdan con los reportados por el CIMMYT (2005).

\section{CONCLUSIONES}

La pudrición de la raíz y tallo del maíz que afecta los campos de maíz «choclo» en la zona estudiada se debe principalmente a Fusarium moniliforme y, en algunos casos, en complejidad con otros hongos como: Exserohilum turcicum, Nigrospora oryzae, Rhizoctonia solani, Phoma sp., Penicilliums sp. y Bipolaris maydis.

Fusarium moniliforme, es diseminado a través de las semillas de donde se inicia la infección de las partes aéreas de la planta.

Los factores que favorecen la severidad de la pudrición de la raíz y tallo del maíz principalmente son: alta humedad, falta de semilla certificada, falta de rotación de cultivos.

Otros factores por los cuales la pudrición de la raíz y tallo del maíz aparentemente se ha vuelto incontrolable en el Callejón de Huaylas, están relacionados a que los agricultores no practican la rotación de cultivos (monocultivo del maíz), aplican productos inadecuados y que la aplicación es inoportuna para el control o manejo de esta enfermedad.

\section{REFERENCIAS BIBLIOGRÁFICAS}

Agrios, G.N. 2002. Fitopatología. México D.F: Editorial UTHEA.

Barnett, H.L. y Hunter, B.B. 1999. Illustrated Genera of Imperfect Fungi. 4ta edition: The American Phytopathological Society.

Durán, R.F. 2007. Control de plagas y enfermedades en los cultivos. Cali: Editorial Grupo Latino Editores. 
Latorre, B. G. 1999. Enfermedades de las plantas cultivadas. 5ta ed. Santiago de Chile: Editorial Universidad Católica de Chile.

Moya, E. y Apablaza, G. 2004. «Identificación de Fusarium moniliforme Sheldon en Semilla de Maíz Dulce». Fitopatología. 39 (1), 33.

Sarasola, A. y Roca de Sarasola. 1975. Fitopatología Curso Moderno. Tomo II. Buenos Aires: Editorial Hemisferio Sur.

Sutton, B. C. 1980. The Coelomycetes. Londres: CMI.

White, Dregor. 2004. Plagas y Enfermedades del maí: Madrid: Mundi Prensa.

Fecha de recepción: 21 de enero 2016

Fecha de aceptación: 27 de mayo 2016

\section{Correspondencia}

Milly Minaya Huerta

sumi2304@hotmail.com 